\title{
The still persistent stigma around diabetes: is there something we can do to make it disappear?
}

\author{
Yasuko Uchigata ${ }^{1}$
}

Received: 15 August 2018 / Published online: 3 September 2018

(c) The Japan Diabetes Society 2018

Stigma is interpreted to mean a negative mark or representation that indicates disgrace and shame, which some individuals are branded with by others or by social groups. Chronic disease, to greater or lesser extents, may evoke feelings of stigma. Regardless of whether or not a person has any individual responsibility in causing a disease, people with a chronic disease may experience a sense of unfairness and feelings close to anger at the fact that they, and not others, have been stricken with chronic disease.

Stigma related to chronic diseases is more often than not closely related to the visibility of the condition or management of the disease $[1,2]$. This leads to self-consciousness about the disease and, in excessive levels, this can cause the individual to distinguish him- or herself from others as being set apart from other people. The individual may also be labeled by others as a person with a disease and viewed as being different. If the person feels that it is easier to simply accept the situation than to resist it, then the individual reaches a state of being indifferent to both others and himor herself leading to deteriorating self-esteem in the person with the disease.

Among chronic diseases, patients with diabetes mellitus, whether type 1 or type 2 , do not appear different from others and can lead a regular life in society, However, diabetes is characterized by the need for the individual to manage the disease by him- or herself with regular, ongoing dietary efforts and exercise regimens in daily life that must be followed to a certain degree continuously, and for which the individual must take responsibility, throughout nearly their entire life, under unending medication or insulin treatments. The individual's regular self-management of the disease is evaluated based on measurements of blood glucose and hemoglobin A1c (HbA1c) levels and the values related to

Yasuko Uchigata

uchigata.dmc@twmu.ac.jp

1 Tokyo Women's Medical University Medical Center East, 2-1-10, Nishiogu, Arakawa-ku, Tokyo 116-8567, Japan lipid metabolism when the individual is examined at the medical institution. Weight and blood pressure measurements are also part of the evaluation. If a poor evaluation continues for many years, diabetes complications with a huge impact on quality of life may develop.

Type 1 diabetes, in which insulin injections are essential, may be seen as a prime example of this described above. No one can be blamed for the onset of type 1 diabetes, which in most cases is explained by the autoimmune mechanisms of the individual. Insulin therapy is started from the time the disease develops. The individual is given an explanation about standard treatment and how blood glucose levels are affected by diet and exercise as well as ways for dealing with those effects, together with standard, textbook insulin therapy. This is done mainly during inpatient treatment. Then, after leaving the hospital, each patient with diabetes, through trial and error, develops his or her own ability to manage glycemic control in his or her own daily life. This is the same whether the insulin therapy is done with multiple injections or with pump therapy.

Unlike Europe and the USA, Japan has a low incidence of pediatric patients with type 1 diabetes and so there are few pediatric patients with type 1 diabetes. Even though they are children, diabetes makes people suspect that the cause was overeating and one often hears of cases in which other people, assuming type 2 diabetes, ask the child if he or she should be eating so much when in fact they are already consuming a normal diet. The ability to give oneself insulin injections, even in the early years of elementary school, is just part of the regular course of treatment for diabetes specialists, but in the mass media, or generally, it is looked upon with astonishment even today.

Given these circumstances, patients with diabetes who need insulin therapy avoid others when performing insulin therapy. When eating together with others, they worry about where they can give themselves an insulin injection. They do not eat the things they like and as they would want, but rather think about how much of the three major nutrients they eat 
and give themselves insulin injections while considering the number of units in the injection. Even the timing of meals is an important point for consideration. People find their own patterns and so become accustomed to adjusting the number of insulin units, but they do not decide the number of injection units themselves. Children with diabetes, who do not know how much of which foods they can eat, must be taught by the parents each time to determine the number of insulin injection units for controlling their blood glucose level, the foods they can eat, and the amount of exercise they must do. In any event, having to do things differently from others three or four times each day outside the home may be the disease self-management of type 1 diabetes. With an awareness of oneself as different from others, the occurrence of stigma would seem normal.

It has been said for many years that such hardship in life is due to the lack of social cognition of type 1 diabetes. The thought has been that the lives of type 1 diabetes patients would become easier if society understood disease management by the individual.

The Japan Association for Diabetes Education and Care and other groups involved in diabetes education in the community have made many efforts to raise social awareness of type 1 diabetes, and these assiduous efforts are continuing today. For example, the revelation of a famous athlete having type 1 diabetes presented a great opportunity to raise social awareness about the disease. Of course, much work in this area still needs to be done.

The author works on the insulin care support committee of the Japan Association for Diabetes Education and Care, which has proposed the introduction of a summer camp for children with diabetes. However, despite our efforts to spread awareness about the possibility of this summer camp to all family physicians treating pediatric diabetes patients, there are still young patients who have not yet been informed. The very small number of pediatric patients with diabetes may also result in a lack of interest among elementary and junior high school teachers. The fact that disturbance of consciousness during school hours due to low blood glucose may be the only point emphasized to many people may also be a factor that creates difficulties for children with diabetes in school life.

Other aspects of difficulty faced by individuals with diabetes trying to lead normal lives in society include dealing with uneasiness when measuring blood glucose in front of others when the individual wants to know his or her blood glucose level, or the discomfort of giving oneself an insulin injection in front of others before a meal.

Even if social awareness is not raised, most people believe it would still be a huge benefit to the individual if he or she did not have to feel the need to avoid the eyes of others. There is a significant expectation that this would help to erase the stigma around diabetes. Therefore, the appearance of small insulin pumps on the market was well received, and while the cost of this equipment is considerable, insulin injections may now be performed discreetly. This was followed by the launch of devices that enabled continuous glucose monitoring (CGM) as well as other convenient devices that automatically perform self-blood glucose monitoring. Sensor augmented pumps (SAP) that act as both an insulin pump and a CGM device have also become available. More recently, a method called flush glucose monitoring (FGM) has become widespread whereby a CGM device is not necessary, and individuals are able to more easily read and understand their own daily blood glucose profile.

I would like to mention a recently reported online survey conducted through social media by the McGill University Health Center in Canada. This survey was on the stigma of bias, discrimination, and shame caused by type 1 diabetes [3]. Canada has a greater number of patients than Japan, but even so, a lack of understanding, discrimination, and bias exist around individuals with type 1 diabetes patients. The authors viewed this as a problem and so planned the study. The subjects were 380 type 1 diabetes patients aged 14-24 years (men 31\%, mean duration of disease 9.6 years, mean $\mathrm{HbA} 1 \mathrm{c} 7.8 \%$ ) in Canada, where nearly $60 \%$ of patients use insulin pumps. About $2.5 \%$ of all respondents self-reported experiencing a blood sugar crash (by lower glucose) once or more per week, while $27 \%$ self-reported a severe blood sugar crash once or more per year. The percent using CGM was not given. In the survey results, $65 \%$ of all respondents reported stigma (this is about the same percentage as in the results of a similar large-scale survey [4] conducted in Switzerland of type 1 and type 2 diabetes patients with a mean age of 67 years old). Many of those reporting stigma were in the high HbA1c group (odds ratio $3.05)$. The number was significantly higher in the group with frequent severe hypoglycemia (odds ratio 1.86) (even after adjusting for sex, age, and insulin administration method). The percentage of women reporting stigma was slightly higher, but the effect from use of an insulin pump was not large. Unfortunately, its association with the use of CGM is unknown since subjects were not asked about whether or not they use CGM.

It may have been wrong to have huge expectations that the stigma of type 1 diabetes patients will lessen with advances in the development of devices for insulin administration and self-monitoring of blood glucose. However, it is also too early to conclude that the stigma of type 1 diabetes patients will lessen with progress in social cognition. From a recent survey of 688 patients with type 1 diabetes in Finland, about $30 \%$ of respondents reported hiding their type 1 diabetes at work because of stigma [5]. Being treated with special care is also embarrassing and is associated with hiding a diagnosis of type 1 diabetes. 
It is a fact that the feeling of stigma is closely intertwined with disease management in the chronic disease of diabetes and so it may be important to once again start listening to patients carefully to fully understand the stigma around diabetes.

\section{Compliance with ethical standards}

Ethics policy This article does not contain any studies with human or animal subjects performed by any of the authors.

Conflict of interest The author declares that she has no conflict of interest.

\section{References}

1. Link BG, Phelan JC. Conceptualizing stigma. Ann Rev Sociol. 2001;27(363-38):5.
2. Schabert J, Browne JL, Mosely K, Speight J. Social stigma in diabetes: a framework to understand a growing problem for an increasing epidemic. Patient. 2013;6:1-10.

3. Brazeau A-S, Meranda N, Wright M, Henderson M, Panagiotopoulos C, Pacaud D, Kearns P, Rahme E, Costa DD, Dasgupta K. Stigma and its association with glycemic control and hypoglycemia in adolescents and young adults with type 1 diabetes : CrossSectional Study. J Med Internet Res. 2018;20:e151 (p1-p10).

4. Gredig D, Bartelsen-Raemy A. Diabetes-related stigma affects the quality of life of people living with diabetes mellitus in Switzerland: implications for healthcare providers. Health Soc Care Community. 2017;25:1620-33.

5. Hakkarainen P, Munir F, Moilanen L, Rasanen K, Hanninen V. Concealment of type 1 diabetes at work in Finland. BMJ Open. 2018:8:e19765. 